\title{
Ultrastructural distribution of collagen types I-VI in aging human retinal vessels
}

\author{
G E Marshall, A G Konstas, W R Lee
}

\begin{abstract}
Retinal vessels from freshly enucleated human eyes were classified into three stages of the hyalinisation process. The distribution of collagen types I-VI within the vessel walls was studied ultrastructurally by immunogold labelling combined with the tissue preparation techniques of cryoultramicrotomy and London resin white embedding. Collagen types I, III, $I V, V$, and VI were found in large vessels, types $I, I V$, and $V$ plus small amounts of III and VI in small vessels, and types $I, I V$, and $V$ in capillaries. Hyalinised vessel walls consisted mainly of types I, IV, and VI collagen.
\end{abstract}

The changes which occur as part of normal aging have been documented at the level of light microscopy ${ }^{1}$ and essentially consist of progressive replacement of myocytes by collagenous tissue. This process of hyalinisation was explored at the level of electron microscopy, and this revealed obvious reduplication of the basement membrane-like material and deposition of $65 \mathrm{~nm}$ banded collagen. ${ }^{2}$ The significance of hyalinisation in blood vessels has been explored in terms of neovascularisation. ${ }^{3-5}$ It is a reasonable hypothesis that a change in the ground substance may modify endothelial cell proliferation in the presence of retinal ischaemia and the subsequent release of vasoformative factors. ${ }^{2-5}$ It is therefore important that the new technology of immunogold labelling be applied to the study of collagen types which are present in aging blood vessels, and in this presentation a preliminary outline of the distribution of collagens I, III, IV, V, and VI will be described.

\section{Materials and methods}

Human retinal tissue was obtained from freshly enucleated eyes of eight patients with choroidal melanoma and four freshly enucleated eyes from patients with other disorders (for details see Table I).

TABLE I Clinical details. Indications for enucleation and classification of retinal vascular disease

Departments of
Ophthalmology and
Pathology, University of
Glasgow, Western
Infirmary, Glasgow
G11 6NT
G E Marshall
A G Konstas
W R Lee
Correspondence to: Professor
W R Lee.
Accepted for publication
9 November 1989

\begin{tabular}{llll}
\hline Age & Sex & $\begin{array}{l}\text { Indication for } \\
\text { enucleation }\end{array}$ & $\begin{array}{l}\text { Classification } \\
\text { of retinal vessels }\end{array}$ \\
\hline 81 & F & Secondary glaucoma & A \\
64 & F & CM & C \\
36 & M & CM & A \\
28 & M & CM & A \\
68 & M & CM & A \\
52 & M & CM & B \\
63 & F & CM & B \\
64 & M & CM & A \\
68 & M & Corneal injury & C \\
64 & F & Metastatic carcinoma & B \\
63 & M & CBM & B \\
76 & M & CM & C \\
\hline
\end{tabular}

$\mathrm{CM}=$ choroidal melanoma $. \mathrm{CMB}=$ ciliary body melanoma
The eyes were immersed immediately after enucleation in a freshly prepared fixative solution of $4 \%$ paraformaldehyde plus $0 \cdot 2-1 \cdot 0 \%$ glutaraldehyde in $0.1 \mathrm{M}$ Sorensen's phosphate buffer or cacodylate buffer $\mathrm{pH} 7 \cdot 2$. After 10 minutes the eyes were opened to allow for rapid penetration of the fixative into the eye. A minimum of 10 blocks were dissected out from every level (primary, secondary, and tertiary) of the vascular tree with regions of arteriole-venule crossover points being preferred. Control tissue was taken from the cornea and sclera. The blocks were washed three times in buffer ( 5 min) and allocated for cryoultramicrotomy, London resin (LR) white embedding, and processing in Araldite for conventional electron microscopy. LR white is a hydrophilic resin that can be used for immunocytochemical studies owing to its property of preserving tissue antigenicity.

\section{CRYOULTRAMICROTOMY}

The blocks were infused in $2 \cdot 3 \mathrm{M}$ sucrose for at least two hours at $4^{\circ} \mathrm{C}$, mounted on cryo pins and snap frozen in liquid nitrogen. Ultrathin frozen sections were cut at $-85^{\circ} \mathrm{C}$ on a Reichert Jung FC4D cryoultramicrotome (Cambridge Instruments) and transferred to 200 mesh carbon Formvar composite nickel grids by means of a $2.3 \mathrm{M}$ sucrose drop and stored on Sorensen's buffer at $4^{\circ} \mathrm{C}$ until commencement of the immunolabelling procedure.

\section{PLASTIC EMBEDDING}

The blocks allocated for LR white embedding were prepared by the following steps, all at $4^{\circ} \mathrm{C}$ : quenching in $0.5 \mathrm{M} \mathrm{NH}_{4} \mathrm{Cl}$ in Sorensen's buffer for at least two hours, washed twice in buffer, dehydrated in two one-hour changes of $70 \%$ alcohol and infiltrated with two one-hour changes of LR white hard grade resin, and polymerised at $50^{\circ} \mathrm{C}$ for 24 hours in size sealed gelatin capsules. Gold coloured ultrathin sections were cut on an LKB Ultrotome Nova (Cambridge Instruments), mounted on 200 mesh nickel grids, and left to dry for $10 \mathrm{~min}$ before storing on $50 \mathrm{mM}$ Tris buffer $\mathrm{pH} 7 \cdot 2$ at $4^{\circ} \mathrm{C}$.

\section{IMMUNOCY TOCHEMISTRY}

The antibody dilutions in Tris buffer plus $1 \%$ bovine serum albumin (BSA) determined by previous experimental work, were 1:40-1:150 for types I, II, III, and V and 1:120 for type IV. The antibodies used against these collagens were raised in goats against both human and bovine collagen and were supplied by Southern Biotechnologies Inc. (UK distributors: Bionuclear 
Services Ltd). Rabbit antibodies against type VI collagen were supplied by Heyl, West Germany, and were used at concentrations between 1:25 and 1:600.

The precise immunocytochemical procedure has been described by Konstas et al. ${ }^{6}$

\section{CONTROLS}

Normal goat serum negative controls for collagens $\mathrm{I}-\mathrm{V}$ and normal rabbit serum negative controls for type VI collagen (both from Sigma) were concurrently conducted at the same dilutions. Human cornea was used as a positive control for collagen types I, IV, and V, and sclera provided a positive control for collagen types I and III.

\section{Results}

An arbitrary classification was used to distinguish the degree of hyalinisation in venules and arterioles. Owing to considerable variation within an individual eye, retinal vessels were placed into one of three grades of the hyalinisation process by an arbitrary classification by light microscopic examination of toluidine blue stained semithin plastic sections. Category A (Fig la) consisted of what was thought to be ostensibly normal vessels in which normal myocytes were identified. In Category B (Fig lb) the walls were significantly thickened but still possessed several layers of myocytes, whereas category C (Fig 1c) consisted of grossly thickened walls with very few myocytes. Five of the cases studied were placed into category $A$, four into category $B$, and three into category $C$.

In all three categories into which the vessel wall was categorised major vessels were found to contain collagen types I, III, IV, V, and VI.

Figure 1: Retinal vessels in various stages of hyalinisation. (a) artery $(A)$ and venule $(V)$ of normal vasculature (category $A)$. (b) hyalinised artery with thickening of vessel wall (category $B)$. Some of the myocytes (arrowed) are atrophic. (c) grossly hyalinised artery (catergory C). Few myocytes are present and the vessel is surrounded by a thick layer of hyaline like material. (Toluidine blue, $a \times 24$; $b \times 60 ; c \times 60$.)

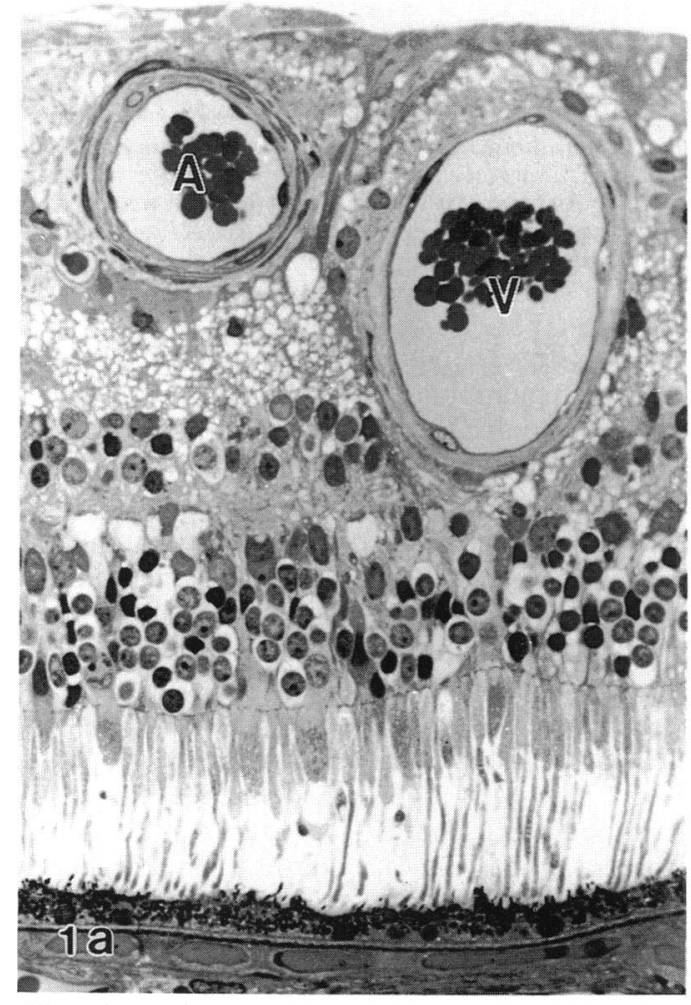

Figure 1a
Smaller branch vessels contained types I, IV, and $\mathrm{V}$ collagen, with diminished amounts of types III and VI. The connective tissue round capillaries contained types I, IV, and V only. Types III and V collagen were found in normal and hyalinised vessels. However, the thickening of hyalinised vessel walls was found to be due to excessive amounts of type I, IV and VI. Type II collagen was consistently absent at all levels of the retinal vasculature.

Type IV labelling was restricted to basement membranes round myocytes and lining the basal aspect of endothelial cells in larger vessels. There was no detectable difference in the intensity of labelling of basement membranes sandwiched between endothelial cells and subendothelial myocytes with basement membranes in the outer part of the vessel wall. The basement membranes of retinal cells that were in contact with blood vessels such as astrocytes, perivascular cells, glial cells, Müller cells were also labelled for type IV. In diseased vessels marked labelling surrounded activated myocytes but was largely absent from atrophic myocytes (Fig 2). Thickened basement membranes were evenly labelled with type IV, as were the multilayered basement membranes typical of hyalinised vessels (Fig 2). The inner half to three-quarters of hyalinised vessels contained type IV collagen, but the outermost region excluding the basement membranes of surrounding glial tissues was negative (Fig 2). It is noteworthy that the collagens in the outer half of the hyalinised vessel walls were identified as types I, III, V, and VI, and the distribution of types $I$ and $V$ in retinal vessels other than capillaries was identical to that of type IV, except that striated fibrils were also positive and that both collagen types were present throughout the entire thickness of hyalinised vessel walls.

Type III collagen was restricted to striated

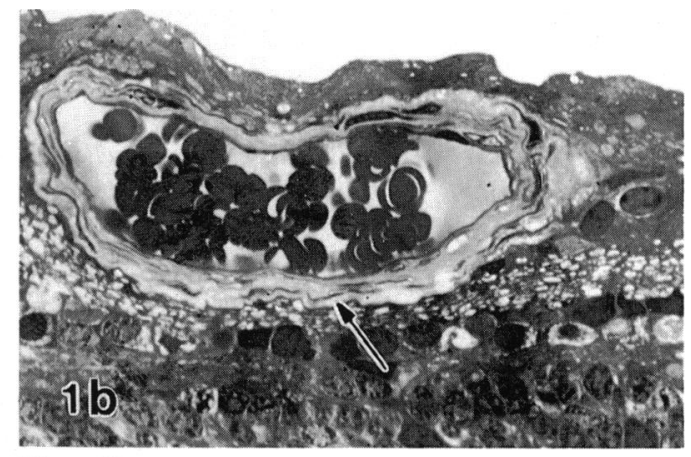

Figure $1 b$

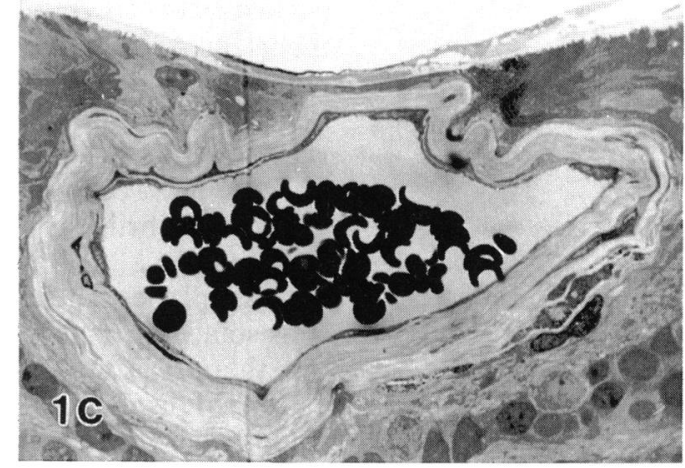

Figure 1c 


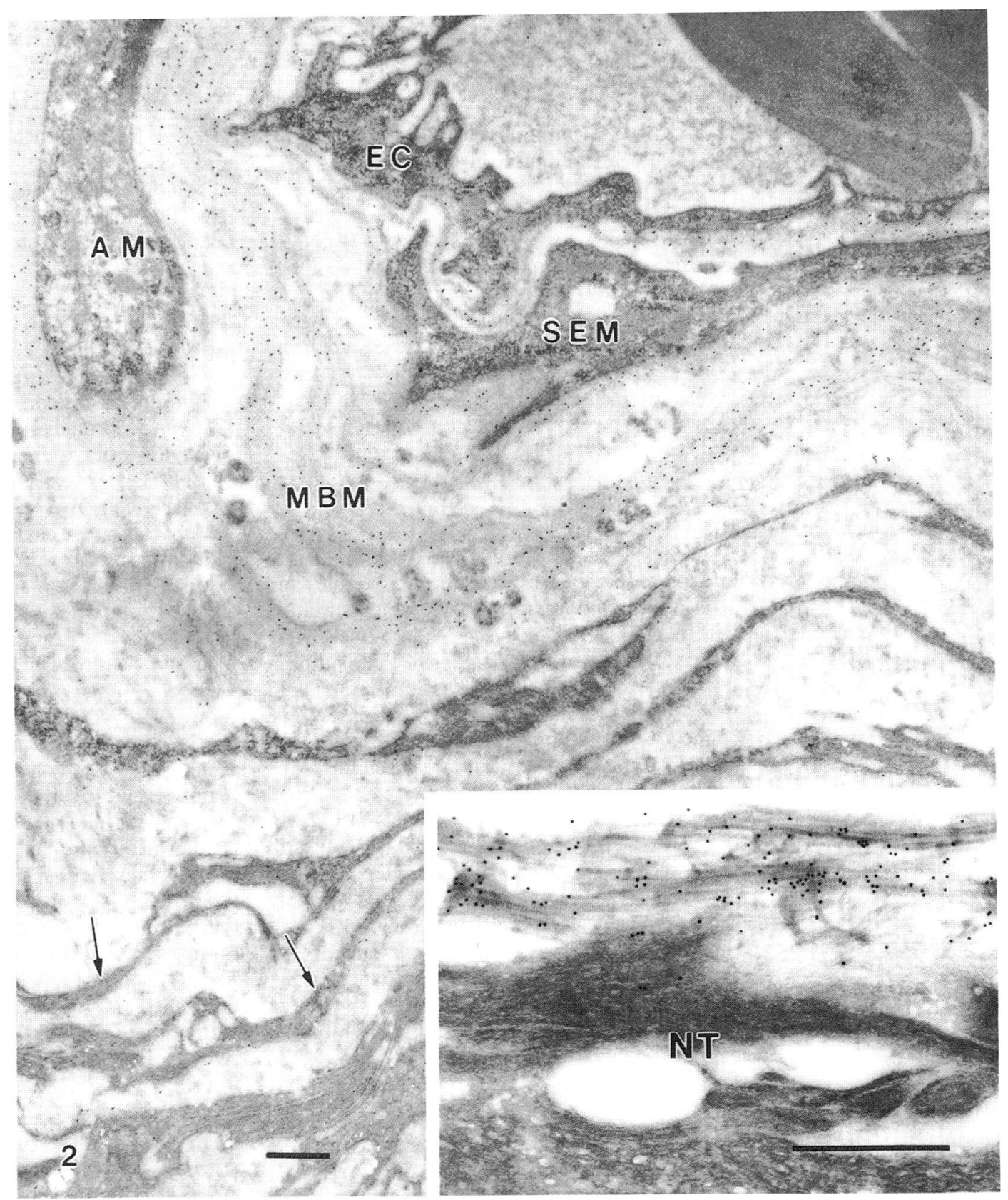

Figure 2: Type IV collagen distribution in hyalinised artery (category $B$ ). The basement membrane of the endothelial cells $(E C)$ and that of the subendothelial myocyte (SEM) are labelled for type IV collagen, as is the multilayered basement membrane $(M B M)$ and an activated myocyte $(A M)$. Atrophic myocytes are void of label (arrowed). ( $L R$ white, $\times 17000, b a r=0.5 \mu m$.)

Insert: Labelling of striated fibrils at outer boundary of vessel wall with type III collagen antibodies. Neural tissue (NT) is free of label. $(L R$ white, $\times 43000$, bar $=0.5 \mu \mathrm{m}$.)

collagen fibrils of large vessels (Fig 2), being completely absent from basement membranes. Type VI labelling was observed on striated fibrils (Fig 3) but was also noted in areas where striated fibrils were absent. Basement membranes sandwiched between endothelial cells and subendothelial myocytes were consistently negative for type VI as well as capillaries and small vessels. In larger vessels immunogold labelling for type VI was present on loose aggregations of collagen fibrils (Fig 3) but was largely absent over areas where the fibrils lay parallel to one another in tight bundles.

In capillaries labelling for type IV (Fig 2) was consistently stronger than that for type I (Fig 4), but there was no detectable difference in the pattern of distribution of the two collagen types. Endothelial cell basement membrane staining for types I and IV was not as intense as that on the outer side of intramural pericyte processes. There was no obvious increase in staining intensity with increasing endothelial cell or pericyte thickness. Capillaries in categories B and C contained a uniform distribution of both types I and IV throughout the width of the thickened basement membrane. Striated fibrils, more clearly visualised in ultrathin frozen sections, were absent.

\section{Discussion}

A detailed search of the literature revealed the absence of studies concerning the fine structural localisation of collagen types in human retinal vessels. Moreover in animal studies such investigations of the retinal vasculature have been limited as to the collagen types examined. Cryoultramicrotomy has not been used in im- 


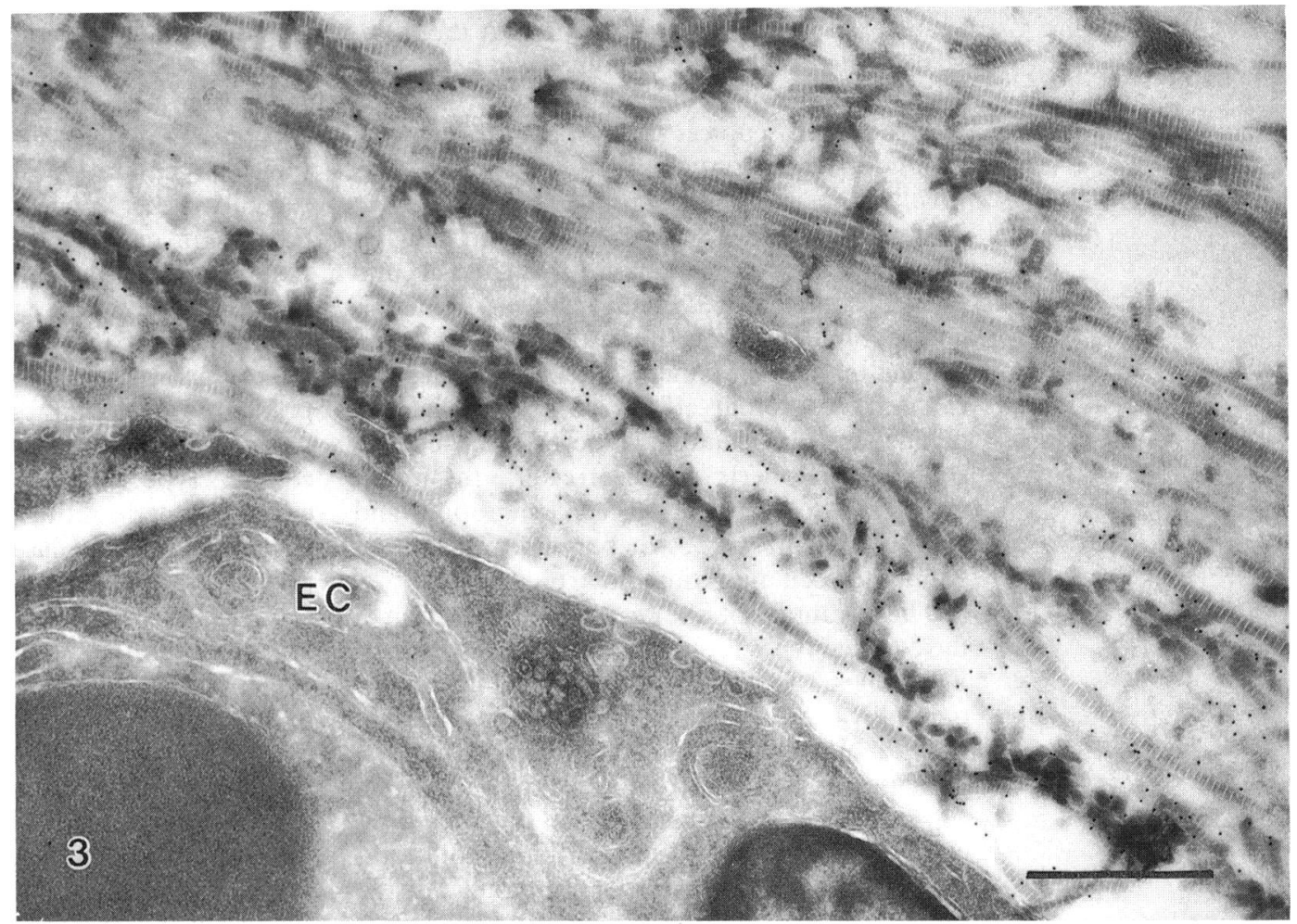

Figure 3: Endothelial cell surrounded by loose aggregates of collagen fibrils labelling for type VI collagen. (Cryo, (44 000, bar $=0.5 \mu \mathrm{m})$.)

munocytochemical investigations of ocular disease, but we have found that with the use of this technique preservation of tissue antigenicity was more satisfactory than that of plastic embedding.

Collagens I, IV, and V have been localised by immunofluorescence in vessels of human, ${ }^{78}$

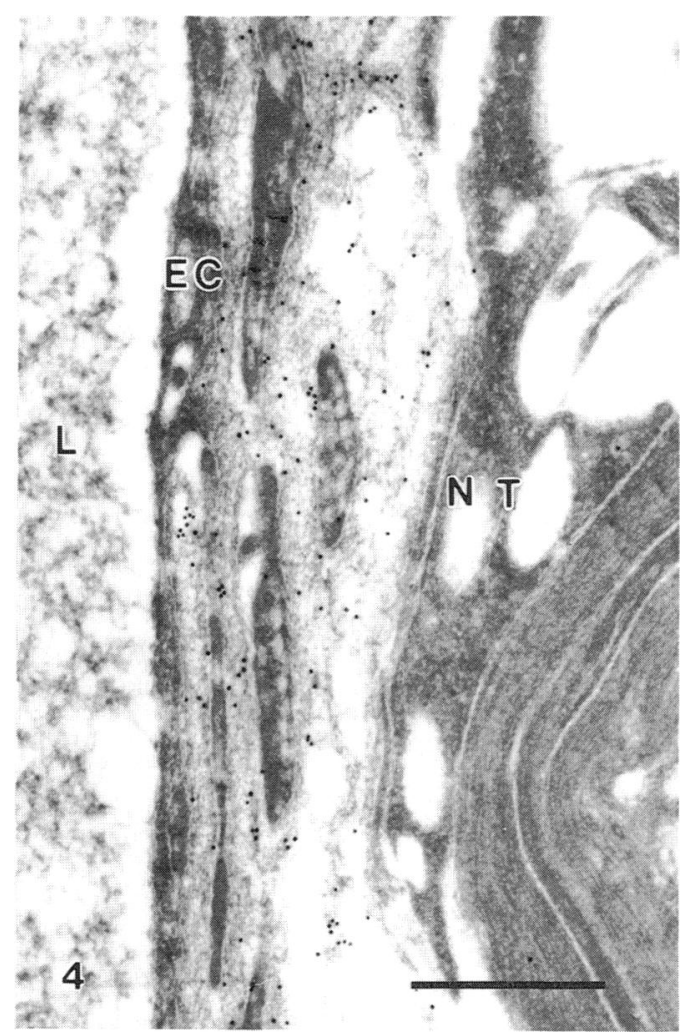

Figure 4: Type I collagen labelling of basement membranes surrounding endothelial cell $(E C)$ and myocytes. Note: vessel lumen $(L)$, neural tissue $(N T)$. (LR white, $(\times 38000$, bar $=0.5 \mu \mathrm{m})$.) bovine, ${ }^{9}$ and rat $^{10}$ retinas. Jerdan and Glaser ${ }^{8}$ found that a broad spectrum of retinal vessels stained for types I and IV, with types III and V primarily staining the walls of larger vessels.

Although immunofluorescence can be used to document the presence or absence of collagen types, the precise distribution within the retinal

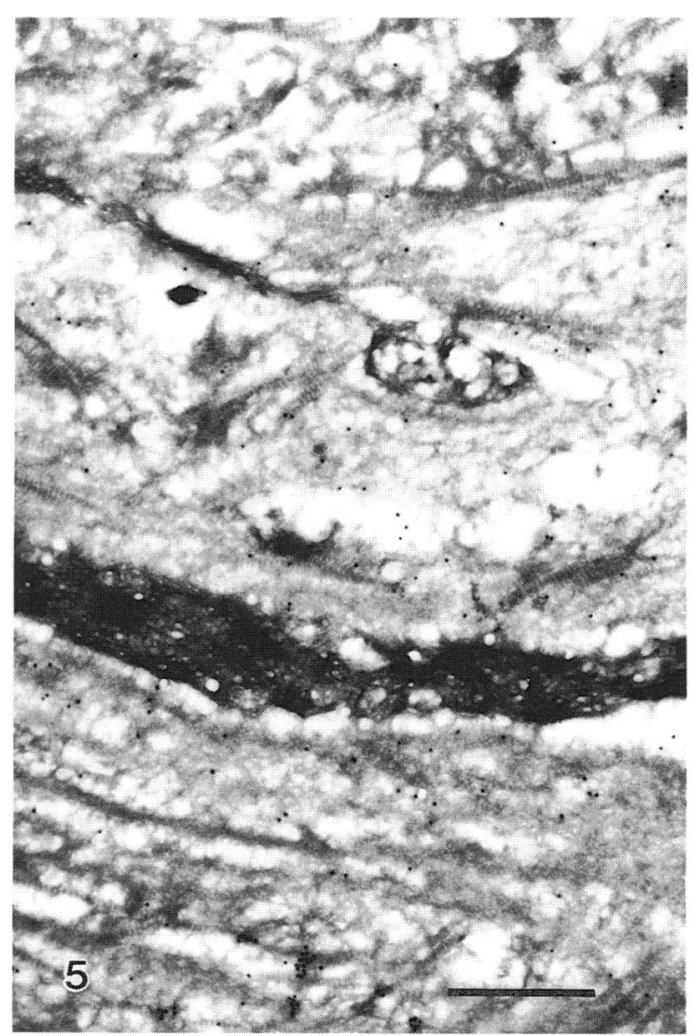

Figure 5: Labelling of vessel wall for type V collagen. Both collagen fibrils and basement membrane are labelled. The tissue separation is attributed to cryo preparation. (Cryo, $(\times 29000$, bar $=0.5 \mu \mathrm{m})$. 
vessel wall is elusive. Information on the ultrastructural distribution of collagen types, made available only through immunocytochemical electron microscopical studies, may be valuable in understanding such phenomena as matrix involvement in intramural neovascularisation. Manschot and $\mathrm{Lee}^{34}$ have emphasised the frequency of neovascularisation within and around the walls of hyalinised blood vessels, and there is considerable evidence that changes in extracellular matrix components, including collagens, can have profound effects on endothelial cell behaviour. For example, type IV collagen was shown by Roberts and Forrester ${ }^{11}$ to inhibit retinal vessel endothelial cell migration and proliferation in vitro, whereas type I collagen stimulated cell migration in vitro.

The advantage of fine structural localisation of the collagen types is underlined in the case of type IV collagen distribution in retinal capillaries. In our study type IV appeared to be more abundant on the outer basal lamina of the intramural pericytes than the basal lamina of the endothelial cells, an observation which could not have been made from the poor resolution immunofluorescent technology. This subtlety in type IV distribution was also noted by Essner and Lin ${ }^{12}$ in rats but had been attributed to failure of antibody penetration. Our results confirm that this is in fact a difference in antigen concentration, since techniques employed in our study eliminate the problem of antibody penetration. This difference in antigen concentration may be extremely significant, since if pericytes produce more type IV collagen than endothelial cells, as this circumstantial evidence would indicate, then such a difference might be of significance in the proliferative stage of diabetic retinopathy. Pericyte loss is an early abnormality in diabetes, and this could presumably lead to a decrease in the amount of type IV collagen, thus removing the inhibiting influence that this collagen has on endothelial cell migration and proliferation.

In immunocytochemistry the use of imunogold is preferred to immunoperoxidase, since with the latter technique oxidised diamino benzidine (DAB) can diffuse from one site to another. This drawback can be illustrated by the report of Abrahamson, ${ }^{13}$ who found that the localisation of laminin obtained with a postembedding technique differed from that seen with the immunoperoxidase technique in glomerular basement membranes in which the label had diffused from the site of antigen location. Immunogold also lends itself to quantitation by image analysis, which has already been performed by other workers. ${ }^{14}$ This may be important in the study of vascular disease, since the relative quantities of various collagen types are probably more significant than documentation of the actual types occurring in the area concerned.

In an immunomicroscope study of extracellular matrix of the monkey optic nerve head using LR white embedding combined with the immunogold technique, Morrison et al's noted the presence of types I, III, IV, and V within the walls of the central retinal artery with only the latter two labelling the basement membranes. Using antibodies supplied from the same source we noted that in addition to types IV and V, type I was also found to be present in the basement membranes of retinal vessels.

It is already known that basement membranes typically contain types IV and V collagen, with several workers demonstrating the presence of type IV in retinal microvessels. ${ }^{816}$ The association of type I with basement membranes as seen in our studies was surprising, as interstitial collagen is not usually found in capillary basement membranes. Nevertheless microvessel preparations from rabbit cerebrum as well as bovine retinal microvessels have been shown to contain type I collagen. ${ }^{16} 17$ On finding this collagen type in retinal vessels, including capillaries, Jerdan and Glaser ${ }^{8}$ suggested that it is the presence of type I collagen that makes the human retinal vasculature so uniquely resistant to trypsin digestion. The extensive $\alpha$-helical structure of type I collagen results in an increased stability to digestion by this protease..$^{18}$

In the present study the fine structural distributions of collagen types I-VI have been documented in normal and aging retinal vessels. The technique of immunogold localisation appears to be of great value in future study of the changes which occur in the extracellular matrix components in ocular vascular disease.

This research was supported by a grant from the W H Ross Foundation (Scotland).

1 Leishman $\mathbf{R}$. The eye in general vascular disease. Hypertension and arteriosclerosis. $\mathrm{Br} \mathcal{F}$ Ophthalmol 1957; 41: 641-701.

2 Lee WR, Blass GE, Shaw DC. Age-related vasculopathy. Eye 1987; 1: 296-303.

3 Manschot WA, Lee WR. Retinal neovascularisation arising from hyalinised blood vessels. Graefes Arch Clin Exp Ophthalmol 1984; 222: 63-70.

4 Manschot WA, Lee WR. Development of retinal neovascularisation in vascular occlusive disease. Trans Ophthalmol Soc UK 1985; 104: 880-6.

5 Manschot WA, Lee WR, Blass GE. Development of neovascularisation in occlusive retinal diseases in adults. In: BenEzra D, Ryan SJ, Glaser BM, Murphy RP, eds. Ocular circulation in neovascularisation. Dordrecht: Martinus Nijhoff/Junk, 1987: 53-6.

6 Konstas AG, Marshall GE, Lee WR. Immunocytochemical localisation of collagens (I-V) in the human iris. Graefes Arch Clin Exp Ophthalmol in press.

7 Newsome DA, Hewitt AT. Laminin detection in normal and retinitis pigmentosa human retina. Cur Eye Res 1985; 4 925-32.

8 Jerdan JA, Glaser BM. Retinal microvessel extracellular matrix: an immunofluorescent study. Invest Ophthalmol Vis Sci 1986; 27: 194-203.

9 Kohno T, Sorgente N, Patterson R, Ryan SJ. Fibronectin and laminin distribution in the bovine eye. $\mathcal{F p n ~} \mathcal{F}$ Ophthalmol 1983; $27: 496-505$.

10 Gordon SR, Essner E. Actin, myosin and laminin localisation in retinal vessel of the rat. Cell Tissue Res 1986; 244: 583-9.

11 Roberts JM, Forrester JV. Factors affecting migration and growth of endothelial cells from microvessels in the bovine growth of endothelial cells from
retina. Exp Eye Res in press.

12 Essner $\mathrm{E}$, Lin W-L. Immunocytochemical localisation of laminin, type IV collagen and fibronectin in rat retinal vessels. Exp Eye Res 1988; 47: 317-27.

13 Abrahamson DR. Recent studies on the structure and pathology of basement membranes. F Pathol 1986; 149: 257-278.

14 Grant DS, Leblond CP. Immunogold quantitation of laminin, type IV collagen and heparin sulphate proteoglycan in a variety of basement membranes. $\mathcal{F}$ Histochem Cytochem 1988; 36: 271-83.

15 Morrison JC, L'Hernault NL, Jerdan JA, Quigley HA. Ultrastructural localisation of extracellular matrix components in the optic nerve head. Arch Ophthalmol 1989; 107 ponents in

16 Kennedy A, Frank RN, Mancini MA, Lande M. Collagens of the retinal microvasculature basement membrane and of retinal microvascular cells in vitro. Exp Eye Res 1986; 42: retinal micr.

17 Faris B, Mozzicato P, Ferrera R, Glenbourtt $M$, Foselli $P$ Franzblau C. Collagen of brain microvessel preparations Microvasc Res 1982; 23: 171-9.

18 Miller EJ, Finch JE, Chung E, Butler WT. Specific cleavage of the native type III collagen molecule with trypsin. Arch Biochem Biophys 1976; 173: 631-7. 